For Consideration In:

Biological Conservation

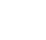

Article Type: Letter to the Editor

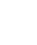

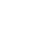

\title{
Analyses of extinction risk are an important part of the conservation process: Reply to Monks
} 8

${ }^{\mathrm{a} A R C}$ Centre of Excellence for Environmental Decisions, School of Botany, University of

Melbourne, Victoria 3010, Australia

${ }^{\mathrm{b}}$ Department of Conservation, PO Box 10-420, Wellington, New Zealand

${ }^{\mathrm{c}}$ Allan Wilson Centre for Molecular Ecology and Evolution, School of Biological Sciences, Victoria

University of Wellington, PO Box 600, Wellington 6140, New Zealand

${ }^{\mathrm{d}}$ School of Biological Sciences, Monash University, Clayton, Victoria 3800, Australia

9 E-mail addresses: david.chapple@ monash.edu (D. G. Chapple), reid.tingley@unimelb.edu.au (R.

0 Tingley), rhitchmough@ doc.govt.nz (R. A. Hitchmough)

2 *Corresponding author. Tel.: +61 399053015

3


It is the sheer number of unknowns that makes conservation a challenging enterprise. Limited information on species' biology, and threats to their persistence, are inherent feature of conservation biology. Thus, conservation efforts generally rely upon expert opinion to fill in the gaps in order to make assessments of extinction risk. For instance, a recent global analysis of the conservation status of reptiles relied on a large international team of experts to develop or review threat assessments using IUCN Red List Criteria (Böhm et al., 2013), a process which involved a degree of subjectivity and reliance on expert opinion.

A similar approach was recently adopted to estimate extinction risk of New Zealand reptiles, using an expert panel and New Zealand Threat Classification System criteria (Hitchmough et al., 2010). The criticisms by Monks (2013) of our recent analysis of the determinants of extinction risk in New Zealand lizards (Tingley et al., 2013) is therefore a generic comment on potential limitations of extinction risk analyses in general, rather than a critique of issues specific to our study. Our study built upon preliminary analyses conducted by Hitchmough et al. (2010), of which Monks (nee Hoare) was a co-author. Importantly, Hitchmough et al. (2010) used the same 2009 threat rankings and examined similar correlates of extinction risk (e.g. body size, habitat use, exotic predators). However, our study was based on a more appropriate analytical approach and a dataset of intrinsic traits and extrinsic threats that was substantially more comprehensive than that used by Hitchmough et al. (2010), and was developed independently of the information used by the expert panel.

Monks (2013) claims that the expert panel used all four of our key predictors of extinction risk (body size, geographic range size, habitat specialisation, rainfall) when assigning conservation rankings due to a lack of empirical data for many species. However, removing species that were assigned a 'Data Poor' qualifier does not substantially influence the explanatory power of our model, with $R^{2}$ changing from $64.5 \%$ to $60.8 \%$. In addition, Monks' (2013) assertion is also 
misleading. Firstly, our study was the first to update the taxonomy of the herpetofauna atlas, and thus our estimates of geographic range size were more refined and up-to-date than those used by the panel. Our original analyses also controlled for the potential circularity of including range size as a predictor by excluding species that were assigned a conservation ranking based on their geographic range. Importantly, this did not alter our general conclusions (Tingley et al., 2013). Secondly, the expert panel did not specifically consider habitat specialisation, apart from the use of broad categories (i.e. generalists vs. specialists). In contrast, we used GIS layers to calculate the number of habitat types occupied by each species after correcting for geographic range size. Thirdly, Monks (2013) claims that the panel considered habitat loss in their deliberations, and thus indirectly considered rainfall because this factor may correlate with historical land use change. However, we included human population density and the extent of habitat loss in our analyses. Thus, the influence of rainfall is likely to extend beyond land use change, and it is incorrect to suggest that the panel considered rainfall in assigning conservation statuses. Accordingly, we argue that the expert panel did not use our four main predictors in their deliberations (at least not at the same level of detail as in our study). In addition, Monks (2013) ignores other important variables that were not included in the highest-ranked model (e.g. human population density, exotic predators, activity) that provide novel insights into the threats facing New Zealand lizards.

We argue that our study helps, rather than hinders our understanding of extinction risk, and plays an important role in the conservation process. Our study was not the final say on the issue, but rather a stage in a feedback loop that is central to the conservation process. Using our model, we highlighted 12 species that were predicted to have a higher extinction risk than that assigned by the panel, and predicted initial conservation rankings for eight data deficient species. The updated 2012 conservation rankings for New Zealand lizards (Hitchmough et al., 2013) were released after the publication of our study and, where changes occurred, our predictions were correct in 50\% (4/8) of 
74 instancesMonks response confirms that our study was completed independently of the panel). For

75 the remaining species, our model was more conservative than the panel, and under-predicted shifts

76 in threat status by a single category for 3 of 4 species. Thus, even if Monks (2013) is correct in

77 stating that our study only "summarizes the rationale of the expert panel", our model still provides a

78 quick and reliable way to assign a preliminary threat ranking for newly discovered or described

79 species. In contrast, Monks' (2013) proposal to use a meta-analytical approach based on population

80 trend data is good in theory, but would be expensive, time-consuming and take decades to achieve,

81 particularly given the limited knowledge of most threatened lizards in New Zealand. Does our study

82 on extinction risk in New Zealand lizards hinder our understanding of the threats to this group or

83 their conservation? No, it represents an important and informative step in the feedback loop that

84 constitutes the conservation process.

85 